\title{
Neuroevolutionary Autonomous Surface Vehicle Simulation in Restricted Waters
}

\author{
A.F. Ayob, N.I. Jalal, M.H. Hassri, S.A. Rahman \& S. Jamaludin \\ University of Malaysia, Terengganu, Malaysia
}

\begin{abstract}
Safe, accurate, and predictable autonomous systems in marine vehicles are paramount. An understanding of an intelligent system fitted inside a ship is critical to ensure an autonomous ship is safe to be operated. Although the use of artificial intelligence in the design of the road-based vehicle has arrived at the self-driving level, there exists a significant gap within the research of autonomous ship to operate in restricted water (riverine and ports). Hence, this article shall discuss the relevant works of literature to set a preliminary guiding principle for the design of an autonomous ship. We present a simple illustrative framework as a starting point for ship designers to begin working in a simulated environment, which can be used as a foundation before the physical autonomous-ships are constructed and tested in a real-world situation. The framework consists of a virtual 3D environment and a surface vehicle with distance sensors, controlled by a neuroevolution-based autonomous piloting system. In this work, two scenarios will be presented: navigation in restricted waters, and obstacle avoidance capability of an autonomous ship. Results show that the resulting autonomous surface vehicle (ASV) is also capable of performing obstacle avoidance in the test track, albeit not being trained to do so in the training track. The work demonstrated in this paper is useful to the ship designers and can be extended for scenario-based planning for autonomous ship design.
\end{abstract}

\section{INTRODUCTION}

Restricted water operations (e.g. rivers and ports) require an autonomous boat to be capable of fast response to avoid obstacles while maintaining its course. A well-designed autonomous boat offers solutions that contribute towards safer waterways and higher economic yields. Such activities include periodically patrolling the river for reconnaissance, environmental monitoring, and transportation of humans and goods. Currently, autonomous boats rely heavily on several hardware devices such as global positioning systems (GPS) [1], distance sensors [2], radars [3], and cameras [4]. While such hardwares are common for the construction of an autonomous boat, several problems exist such as the high price of radar,
GPS multi-pathing which resulted due to highdensity vegetations, and expensive high-quality cameras which also require frequent maintenance. This leaves the autonomous boat with the use of distance sensors, which may offer a robust [5] and low-cost solution to assist in navigation and obstacle avoidance tasks, driven by a well-trained artificial intelligence system.

Fundamentally, an autonomous boat/autonomous surface vehicle is a subclass of mobile robots. Conceptually, a mobile robot consists of a control system, sensors (distance sensors, light sensors, vision sensors), and actuators (motors, servos) to respond to its surrounding [6]. An autonomous mobile robot is capable of sensing its surroundings and acting upon 
the surrounding based on its mission definition. In the field of maritime technology, there are vast potential use-cases for autonomous surface vehicle robots. While the primary mission of an autonomous surface vehicle varies (e.g. reconnaissance, patrol, intercept, environmental monitoring), several common themes constitute as the sub-tasks of such autonomous surface vehicles such as manoeuvring [7]-[10], cruise control [11]-[14] and collision avoidance [9], [10], [15].

In this paper, we present a concise state of the art 5-years review on the application of neuroevolutionary methods in maritime technology, particularly in the ship design discipline. A fundamental example using state-of-the-art tool is presented, where an autonomous surface vehicle (ASV) or an agent is shown to interact with an uncertain environment (dynamic water response due to buoyancy, uneven riverbank terrain, potential collision situation with other floating objects) while avoiding any collision situation through automated steering and thruster control.

In this work, autonomous control of a surface vehicle is designed where an agent demonstrates river navigation while avoiding obstacles in a dynamic environment, using artificial neural network (ANN), as shown in Figure 1. The desirable steering and throttle control are achieved using ANN in which the weight and biases of the ANN are found using the unsupervised machine learning method. The unsupervised machine learning method used in this work is reinforcement learning, in which an agent is rewarded based on the attainment of accumulated checkpoints number in each simulation run. This is achieved using Genetic Algorithm (GA), in which for every simulation runs, the best designs shall be retained to evolve in a search for the best design until an arbitrary generation number achieved.

We evaluate the performance of the neuroevolutionary ship control using computer simulation, focusing on the ship's capability to follow the river path, while at the same time avoiding collision with other floating objects such as weather buoys.

The main contribution of this paper is the state-ofthe-art review of neuroevolutionary ship control and the experimental results of an autonomous surface vehicle operating in a highly dynamic environment. The review of literature shall be elaborated in the next section, followed by the methodology, experimental results, discussion, and conclusion.

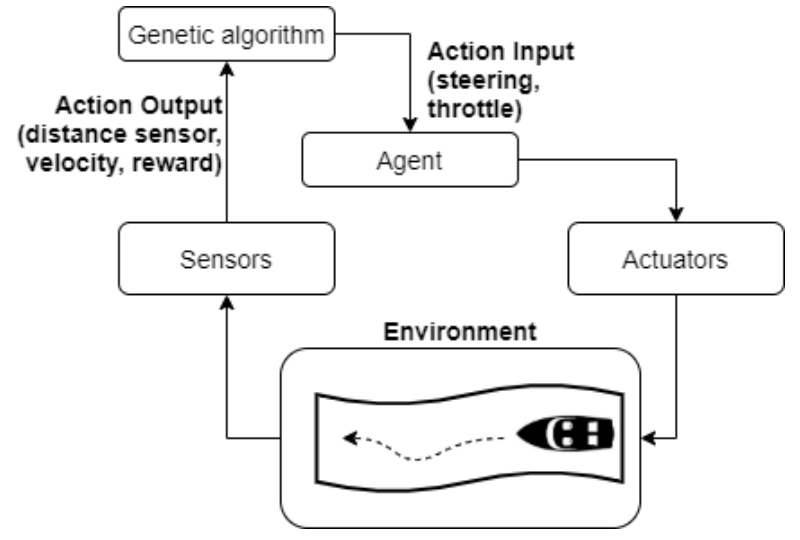

Figure 1. Neuroevolutionary ship manoeuvring control.

\section{RELATED WORKS}

Neuroevolutionary-based works in the literature concerning ship steering or piloting remains lacking as compared with autonomous cars. This is due to the high volume of production of cars compared with ships within consumer markets. Based on the keyword search (Elsevier's ScienceDirect) on research and review articles of 'autonomous car' versus 'autonomous ship', a stark difference (50\% differences) in terms of volumes of literature can be seen, as shown in Figure 2. Such preliminary search has demonstrated a considerable research gap within this discipline and requires attention as the industry is moving toward the $4^{\text {th }}$ Industrial Revolution.

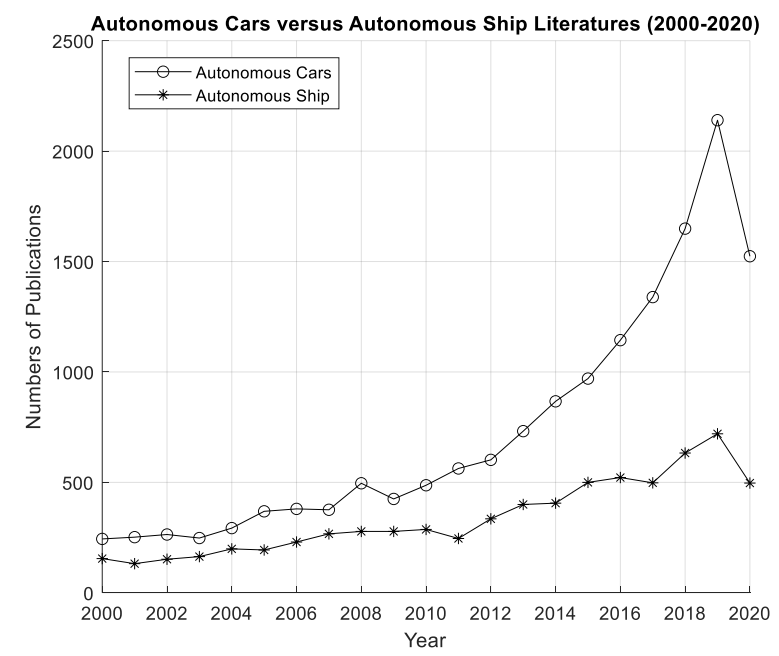

Figure 2. Numbers of publications between Autonomous Cars versus Autonomous Ships spanning across 20 years.

In this section, the related works available in the literature concerning autonomous ship shall be reported. The subsection shall be grouped into three major topics, which are the application of neuroevolution (evolutionary-based artificial neural networks), the study of ship motion of autonomous ships, and finally the safety-related works in autonomous ship developments. 


\subsection{Neuroevolutionary Works in Ship Steering/Piloting}

Autonomous ship berthing study was presented by [16], [17] where backpropagation (BP) neural network was incorporated to study the propeller revolution controller under gust wind disturbances. Through the use of a feedforward neural network, the study was extended in [18], [19], [20] to justify the network's real-time response within the context of safe distance. Similar work using ANN controller for automatic berthing in different ports was presented in [21] and [22]. A neuroevolutionary neural network constructed via the use of evolutionary algorithms was proposed by [23] to perform the task of crossing or overtaking the ship-based COLREGs rules. This ensures the ship to manoeuvre safely and efficiently. Within the same context, [24] compared the use of direct and indirect encoding for neuroevolutionarybased ship handling, which uncovered the ability of indirect encoding to generalize and react to new states without bias towards the learned patterns, however noting that direct encoding method may adapt faster to new sudden changes.

Path following is crucial to maintain the safe cruise of a ship. The works presented in [25] demonstrated the capability of a vessel to return the course-keeping path of a ship on the routes efficiently using the neuroevolutionary method. Reported by [26], such an algorithm is independent enough to find the most effective path by considering a list of possible solutions.

It can be noted that most of the works presented in the field of neuroevolutionary ship piloting are within the scope of simulation works [27]. Ship simulation is a very powerful method to represent real-life situations, to train ship operators and intelligent algorithms while at the same time examining typical scenarios in real-world situations [28].

\subsection{Motion Study of Autonomous Ships}

One of the challenging aspects of control system development in marine discipline is the highly nonlinear motion of ships, compared with road-based vehicles. [7] demonstrated the use of artificial intelligence to steer ships in completing manoeuvring tasks autonomously. A hybrid of fuzzy logic and artificial neural network (ANN) to perform autopilot ship navigation was demonstrated in the work of [8], while [9] proposed the use of a recursive neural network to perform tactical circles and zig-zag motions. An innovative offshore autonomous rescue vessel was introduced in the work of [11], [12] to assist large ships in the search-and-rescue mission.

Radial basis function (RBF) neural network was incorporated by [13] to approximate the unknown non-linear terms of the non-linear ship course control system. Via the use of similar techniques, [14] demonstrated an approach to simulate and control the ship's motion which caters to environmental disturbances. [29] recommended the use of recursive neural networks (RNN) due to its flexibility in counter-measuring ship dynamics where they give useful perspectives for solving the problems in control theory as well as applications in marine systems.

In visual perception systems for autonomous ships, [30] analyzed four artificial intelligent algorithms and deep learning frameworks on the case of object detection and tracking, namely; K-Nearest Neighbor (K-NN), Artificial Neural Network (ANN), Convolutional Neural Network (CNN) and Deep Convolutional Neural Network (DCNN). In view of automatic ship navigation systems with collision avoidance, [31] proposed the use of potential field method in terms of its effectiveness and practical applications. Presented by [32], a Neural Network Allocation (NNA) was used to provide the transformation between the desirable generalized forces as input and the individual thruster command for the output.

\subsection{Safety-Related Works}

Convention on the International Regulations for Preventing Collisions at Sea (COLREGs) is essential to apply for the manned and unmanned vessel. To comply with COLREGs rules, [10] presented the application of neuroevolutionary methods in vessel overtaking, head-on, and crossing scenarios using artificial potential field (APF). However, [33] argued that COLREGs regulation might fail in some instances when both of the ships decide to take the same decision, which may lead to ship collision. Therefore it is suggested that any two vessels; (1) should not be overtaking when there exists a crossing vessel, and (2) an overtaking can be done by a faster vessel when there is no crossing vessel in the area of proximity [23].

A complete collision avoidance system should consider certain factors (e.g. ship types, traffic status, weather, and navigation technologies) [34]. Therefore, to improve the collision avoidance system, [35] proposed a reward function as the main component of Concise Deep Reinforcement Learning Obstacle Avoidance (CDRLOA) to act as a feedback data to the system that evaluates the system performance of the control behaviour at one scalar signal. [36] proposed the dynamic predictive guidance technique to address collision avoidance. It is agreed upon that collision avoidance should meet the following optimal rules; collision avoidance's safety and network availability, and the shortest navigation distance during collision avoidance [37].

\section{NEUROEVOLUTIONARY-BASED SHIP STEERING/PILOTING}

In this section, the methodology for neuroevolutionary-based ship steering control shall be presented. The basic concepts of genetic algorithm shall be discussed, followed by the description of agents (autonomous surface vehicle, ASV) and finally the description of both the training track and testing track incorporated in this work. 


\subsection{Description of Neuroevolutionary-based Ship Steering Control}

An artificial neural network is considered as an individual in a population generated by Evolutionary Algorithms (EA) such as Genetic Algorithms (GA). Each ANN can vary in terms of its structures, or its weights and biases, which are incorporated into each of the individual agents within an environment. In this work, a Conventional Neuroevolution (CNE) is incorporated, in which the sets of weights inside of a fixed-structure ANN are evolved using GA [38] [39] [40]. Such an environment which is being utilized as a platform to investigate the response of the agent can be set up in two ways, whether in a physical environment or virtual simulations. The response of the agents (e.g. distance to a potential collision, the velocity of agents, hydrostatic/hydrodynamic performance, distance to a target, current position of the agent) can be used as the input (objective functions, variables, constraints) for the EA, in which assisting in providing for the next generation of the population.

The pseudo-code of the CNE-based neuroevolution case study for an autonomous mobile robot operating on a water surface can be observed in Algorithm 1 below. In this case study, the structure of the ANN is fixed with input with the size of 10 neurons (9 distance sensor inputs and one velocity input), a hidden layer with the size of 3 neurons, and an output layer with the size of 2 neurons (turning angle coefficient, thruster coefficient). Using the fixed structure of the ANN, an initial set of weights and biases are generated randomly which are assigned to each of the agents $(P(g=1))$. Upon receiving the neurons, the agent shall complete its task to maximize the number of checkpoints (reward) by autonomously piloting itself within a challenging test track. In a situation where the agent collided with the riverbank of the track, the agent shall be discarded (penalty) for that generation $(g)$. The following generation shall continue where the offspring $\left(P_{c}(g)\right)$ are generated from the best agent from the previous generation until the stopping criteria (e.g. number generations) of the GA is achieved.

Algorithm 1. Pseudo-code for the genetic algorithm in the neuroevolution case study

$1 \mathrm{~g}=1$;

2 initialize $\mathrm{P}(\mathrm{g}=1)$

3 while isNotTerminated() do

evaluateFitness $\mathrm{P}((\mathrm{g}))$;

sortFitnes $\mathrm{P}((\mathrm{g}))$;

$\mathrm{P}(\mathrm{g})=$ selectIndividual $(\mathrm{P}(\mathrm{g}))$;

$\mathrm{P}(\mathrm{g})=\operatorname{cross}$ Over $(\mathrm{P}(\mathrm{g}))$;

$\mathrm{P}(\mathrm{g})=$ mutation $(\mathrm{P}(\mathrm{g}))$

$\mathrm{P}_{\mathrm{c}}(\mathrm{g})=$ selectParents $(\mathrm{P}(\mathrm{g}))$

$10 \mathrm{P}(\mathrm{g})=\mathrm{P}_{\mathrm{c}}(\mathrm{g})$; // Children population became the

parents for the next generation.

$11 \mathrm{~g}=\mathrm{g}+1$

12 end

The experiment can be described as a highly nonlinear constrained optimization explained as below:

Objective function: Maximization of the number of checkpoints $f(x)=f\left(x_{1}, \ldots, x_{10}\right)$, where variable $x_{1}$ to $x_{9}$ is the array of distance measured from the riverbank to the body of ASV, and variable $x_{10}$ is the velocity of the ASV during the time of measurement. Each member of the population aims to maximize the number of checkpoints achieved given a prescribed time in seconds.

Constraint: A boolean data of $\{0,1\}$ condition in which the body of the ship should not touch the riverbank or any floating object on the water. If the body of the ship collided, the boolean would be set as \{1\} which deemed unfit and discarded from the remaining population of the agents, whereas $\{0\}$ means the agent is fit in which the fitness shall be recorded for further evaluation of the next generation run.

\subsection{Description of Agents (ASV)}

Autonomous Surface Vehicle (ASV) is a subclassification of Autonomous Mobile Robot, which operates on the water surface. In this work, the ASV is modelled using typical dimensions of workingclass Hydrographic Surveying ASV available in the market [41], shown in Figure 3 and detailed in Table 1. In this work, the ASV is equipped with an array of 9 distance sensors with an interval of $20^{\circ}$, located from the perpendicular of port-side to the starboard, as shown in Figure 4.

The simulations conducted in this work incorporated the commercial mesh-based physics modelling codes [42] to model the ASV buoyancy force (Equation (1)) and hydrodynamic force (Equation (2)) in real-time. The simulation environment is created within the Unity physics engine, which suitable for fast physics modelling (utilizing C\# programming language) in the preliminary design stage. In this work, the ASV shall only rely on the input gathered from the distance sensors and its velocity to decide its value of the thruster coefficient and its rudder angle coefficient.

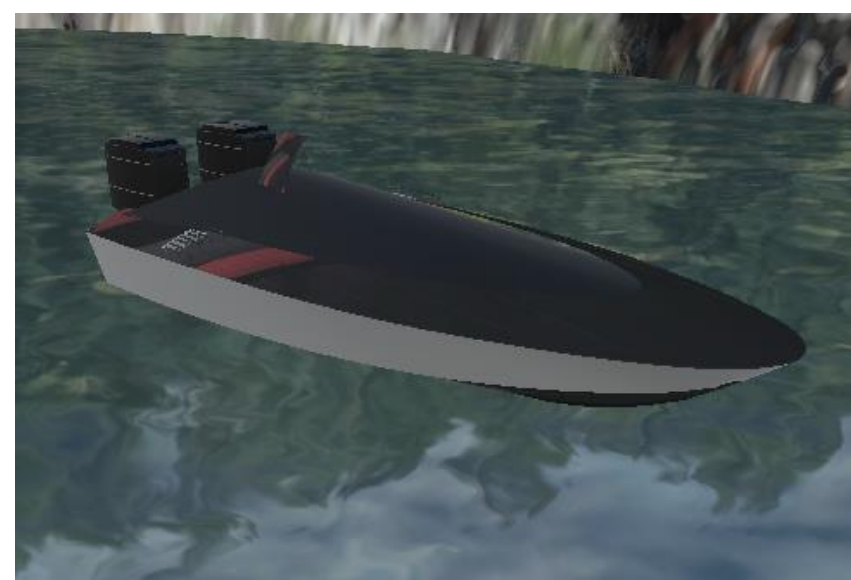

Figure 3. A typical working-class Hydrographic Surveying ASV. 


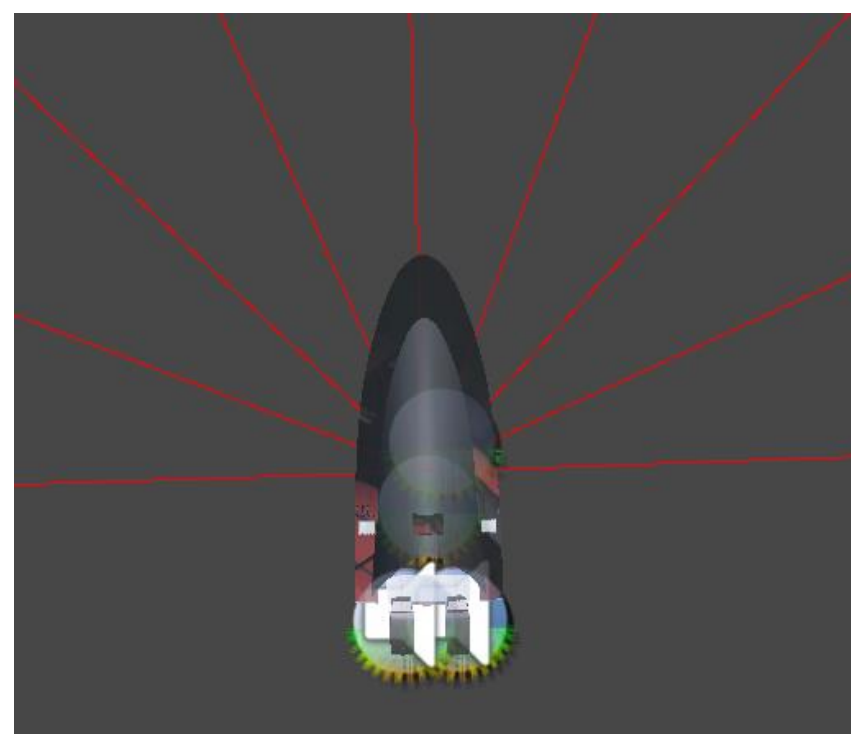

Figure 4. An array of 9 distance sensors with an interval of 20 degrees is incorporated in the ASV.

Table 1. Principal particulars of a working-class hydrographic surveying ASV used in this work

\begin{tabular}{ll}
\hline Particulars & Dimension \\
\hline Length & $2.4 \mathrm{~m}$ \\
Beam & $1.0 \mathrm{~m}$ \\
Draft & $0.3 \mathrm{~m}$ \\
Weight & $500 \mathrm{~kg}$ \\
Top speed & $7 \mathrm{knots}$ \\
Number of sensors & 9 distance sensors \\
\hline
\end{tabular}

$F_{B}=\rho g V$

where,

$F_{B} \quad$ : Buoyant force in $\mathrm{N}$

$\rho \quad:$ Fluid density in $\mathrm{kg} / \mathrm{m}^{3}$

$V \quad$ : Displaced body of fluid in $\mathrm{m}^{3}$

$g \quad$ : Gravity constant, $9.81 \mathrm{~m} / \mathrm{s}^{2}$

$F_{D}=\frac{c_{D} \rho v A}{2}$

where,

$F_{D} \quad:$ Drag force in $\mathrm{N}$

$C D \quad$ : Drag coefficient

$\rho \quad:$ Fluid density in $\mathrm{kg} / \mathrm{m}^{3}$

$v \quad:$ Velocity of ship in $\mathrm{m} / \mathrm{s}$

A : Surface area underwater in $\mathrm{m}^{2}$.

\subsection{Description of Tracks (Training and Testing)}

The training tracks incorporated in the simulation are designed to mimic a very tight and challenging restricted water scenario, as shown in Figure 5. The training track consists of several characteristics, such as:

1 one straight cruise

2 four sharp U-turns

3 one sharp $90^{\circ}$ corner

4 the width of the riverbank is $10 \mathrm{~m}$

5 the dimension of the training track is $60 \mathrm{~m}$ width and $100 \mathrm{~m}$ length

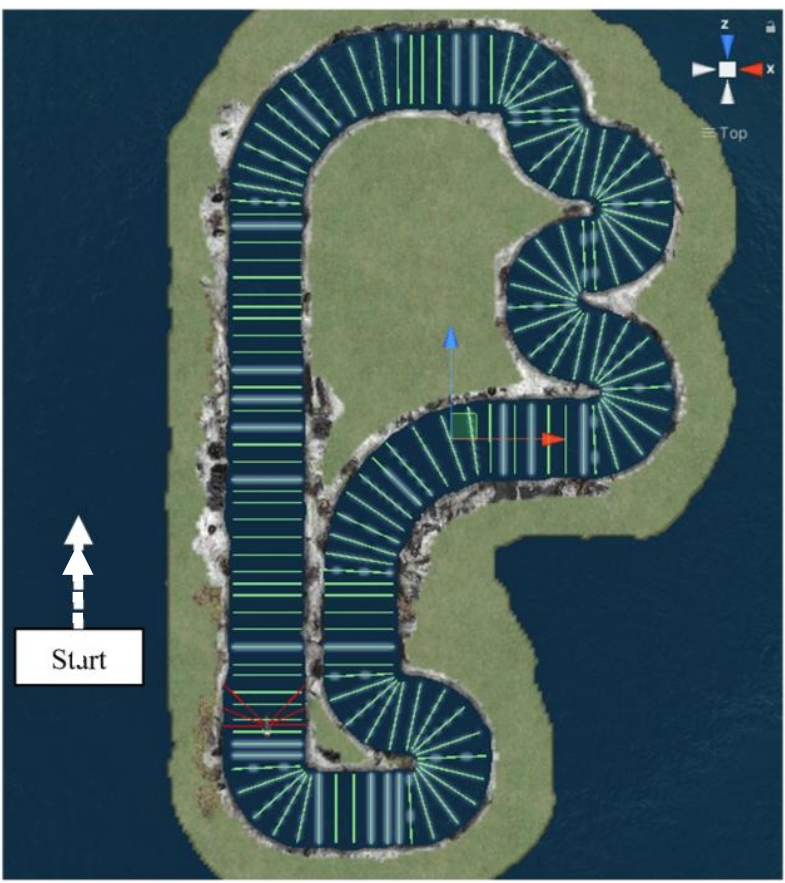

Figure 5. Training track shown from the Top-view, with the 'checkpoints' highlighted.

The testing track incorporated in the simulation is a larger restricted water environment, e.g. an inland water transport scenario, as shown in Figure 6. Surrounded by typical objects in ports (buildings, boats, weather buoys, and deck), the testing track differs in terms of the size of the river with additional obstacles to test the generalization capability of the resulting ANN model. The training track can be summarized as below:

1 three straight cruises

2 six sharp U-turns

3 two sharp $90^{\circ}$ corners

4 the width of the riverbank is $20 \mathrm{~m}$

5 the dimension of the training track is $130 \mathrm{~m}$ width and $140 \mathrm{~m}$ length

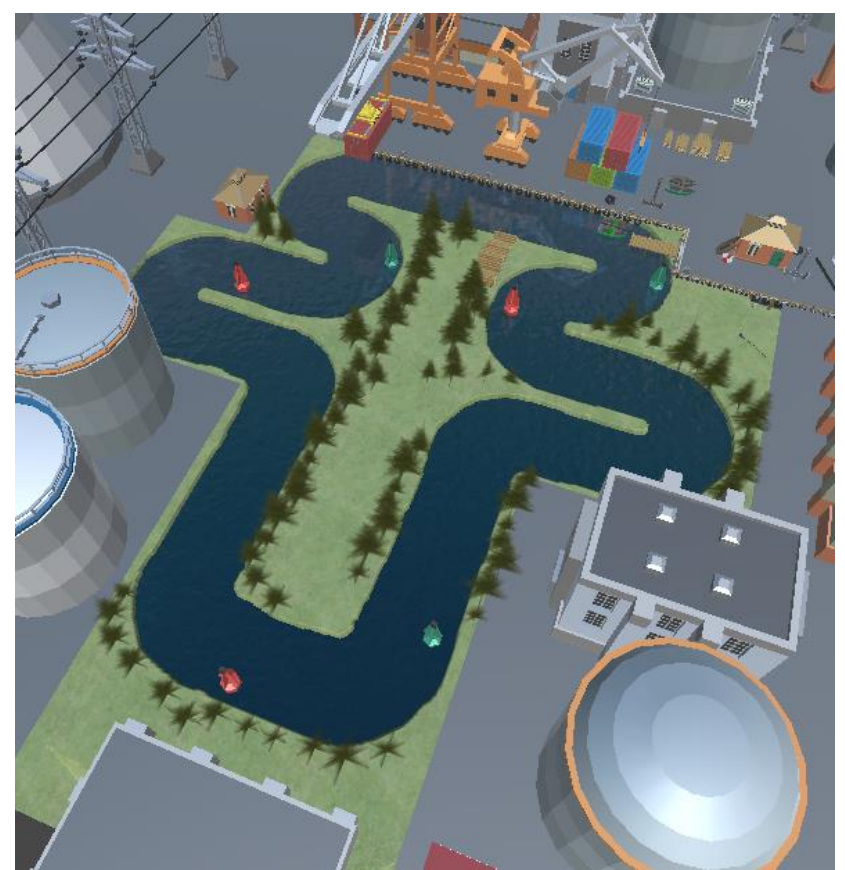

Figure 6. Testing track shown from the Perspective-view, with the buoys and boats floating on the water to represent obstacles. 


\section{RESULTS AND DISCUSSIONS}

In this section, the results that demonstrate neuroevolutionary-based ship steering control shall be presented. The manoeuvring characteristics of the best agent shall be discussed concerning the training track, followed by the observation of the obstacle avoidance capability shown in the testing track.

\subsection{Training Track Autonomous Manoeuvre}

The autonomous manoeuvre of the ASV within the training track is shown in Figure 7. It can be observed that the ASV was able to negotiate turns successfully using the CNE-based ANN. With respect to the manoeuvring characteristics, three sharp corner manoeuvres on the North-East of the training track is highlighted in Figure 8 and highlighted in green colour, together with its respective zone 1, 2 and 3 .

As the ASV cruising from the bottom right (SouthWest) of the training track, it can be understood that the positive angle values of the rudder angle represent the right turn, while the negative rudder angle value represents the left turn. In this experiment, as the ASV cruise via the assistance of distance sensor (input), the ANN model evaluated the sensor input to produce the best throttle percentage and the rudder angle values, as shown in Figure 9.

It can be observed in Figure 8, accompanied by Figure 9, as the ASV enters Zone 1, the rudder tends to turn the ASV to the right to follow the U-turn curve to the right. The same pattern can be observed in zone 3. While manoeuvring against zone 2 , the ASV heads to the left to comply with the left U-turn. In all three zones, it can be observed that the throttle and velocity value spikes up and down significantly each time an aggressive manoeuvre is being executed, which is a desired characteristic for a CNE-based ANN model.

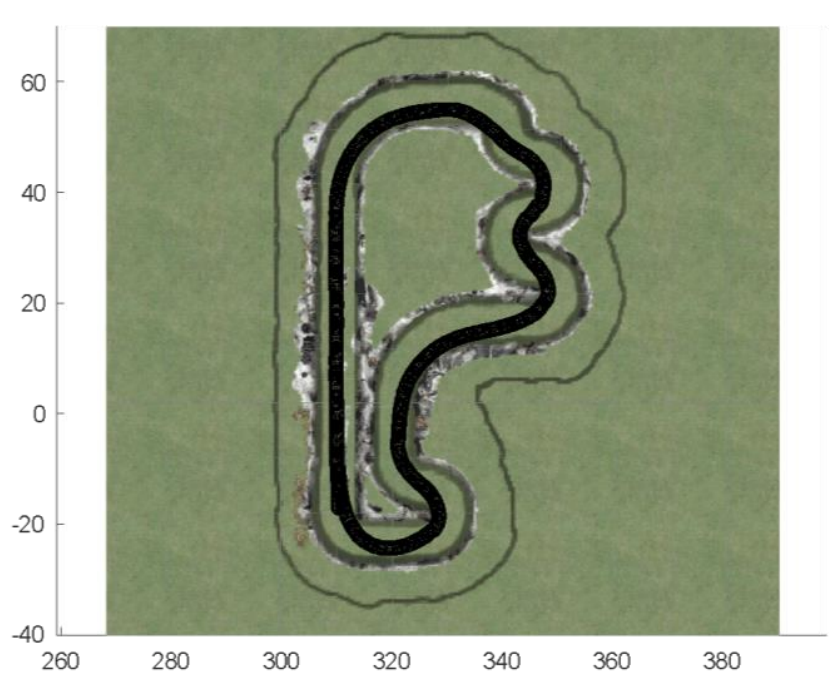

Figure 7. Overall autonomous manoeuvre at the training track with the ASV's path highlighted in black colour.

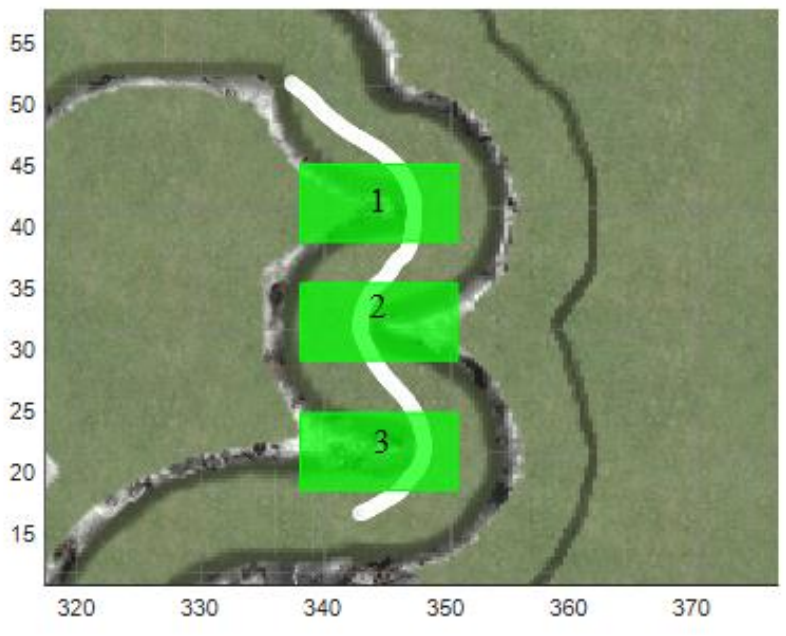

Figure 8. Autonomous sharp corner manoeuvre, highlighted in green colour, with the zones labelled as ' 1 ', ' 2 ' and ' 3 '.
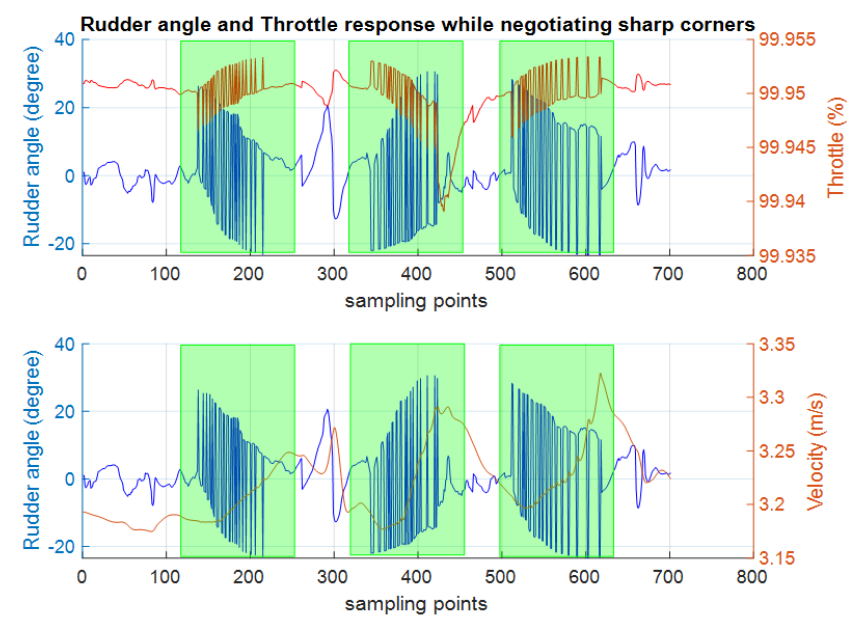

Figure 9. Rudder angle, throttle, and velocity responses while negotiating with sharp corners as highlighted in green colour.

\subsection{Test Track Autonomous Manoeuvre}

In the second experiment, the same ANN model trained in Section A previously was incorporated to investigate the capability of the model to generalize the manoeuvring characteristics in an unfamiliar surrounding using a test track. The top-view of the test track (Figure 6) is presented in Figure 10, depicting the successful autonomous manoeuvre of the ASV using the ANN model. In this section, an additional challenge vis-à-vis the training track is incorporated, where six weather buoys were located on the U-turns and sharp turns, together with two boats and one deck situated on the North-East of the training track.

Depicted in Figure 11, two zones were shown; zone 1 for the boats and deck obstacle, while zone 2 is for the weather buoy obstacle. It can be observed that within both zones, two quick manoeuvres to the right were observed where the ANN is preventing the ASV from colliding with the boats and weather buoy by performing quick rudder turn to the negative angle. It can also be observed that the throttle and velocity values significantly dropped each time an aggressive 
manoeuvre is being executed. It is shown in Figure 12 that the ASV is experiencing steady control of the rudder and speed in between the two zones depicting that no unsteady manoeuvre is being performed in a straight direction cruise.

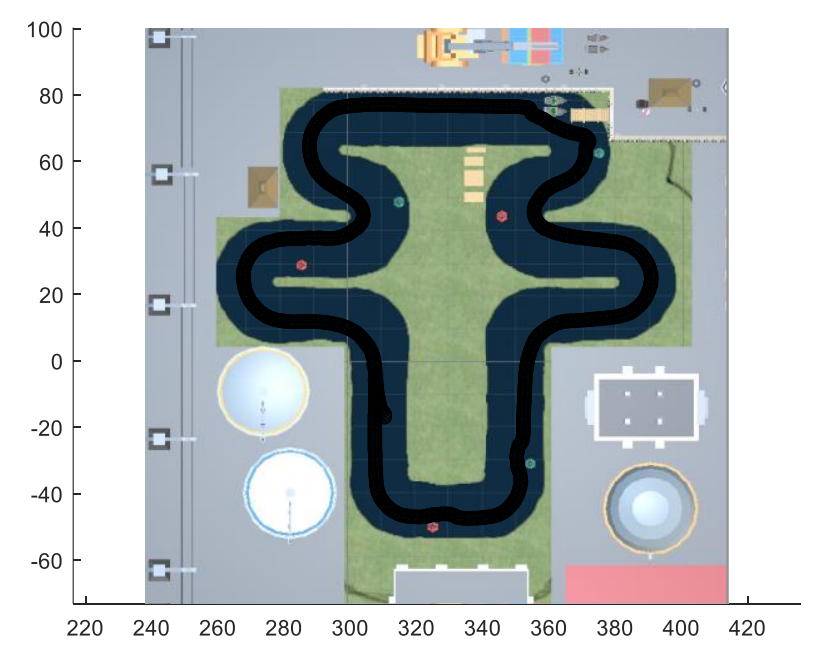

Figure 10. Overall autonomous manoeuvre at the test track with the ASV's path highlighted in black colour.

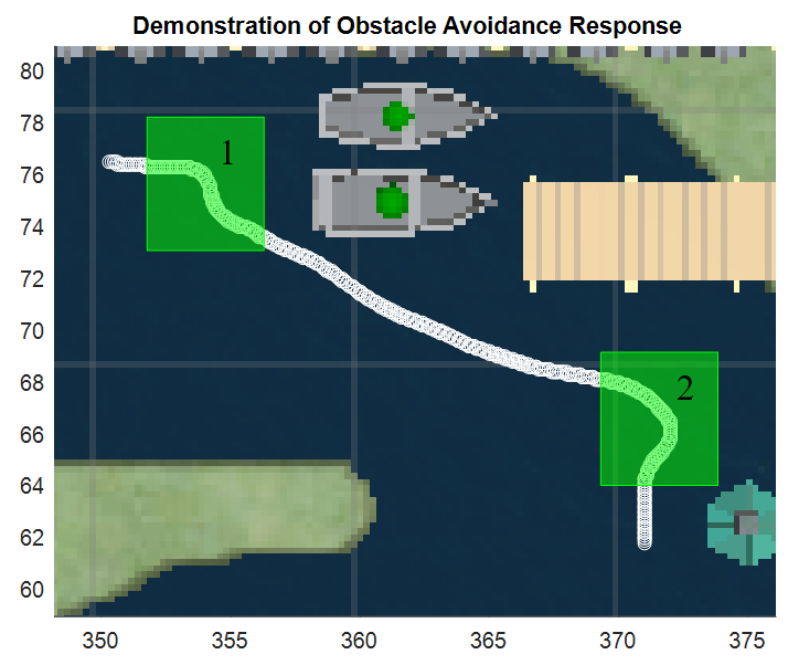

Figure 11. Autonomous obstacle avoidance manoeuvre, highlighted in green colour.
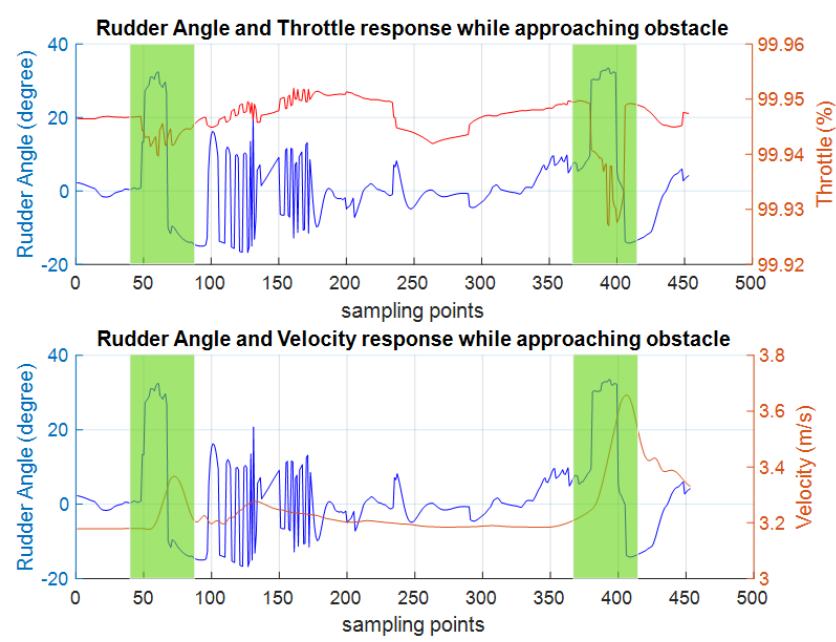

Figure 12. Rudder angle, throttle, and velocity responses while negotiating with an obstacle. Obstacle avoidance is highlighted in green colour.

\subsection{Discussions: Comparative Handling Characteristics} between Human and Autonomous System

The performance of the trained ANN model as evaluated in Section A and Section B has indicated that the ASV is capable of performing safe autonomous cruising, while at the same time avoiding obstacles. Such findings are further strengthened with the observation that the ASV is competent to perform safe navigation in an unfamiliar setting, as evaluated in Section B. While such capability is very desirable in the realm of control, it can be hypothesized that such ASV is capable of performing better than a human operator. Taking into account that the ASV is equipped with nine distance sensors (Figure 4), the data of humancontrolled vessel navigating the training track is recorded (Figure 13) to compare the efficiency of the autonomous system between both human and the ASV.

As depicted in Figure 14, a 50m straight-line cruising operation was captured for 10 seconds are compared between a human operator and the ASV. It can be observed that a human operator who relies on visual perception are more relaxed during the straight-line course-keeping operation. Whereas, for an autonomous system equipped with nine distance sensors, the ASV actively measures the safe distance, which in turn beneficial for active obstacle avoidance. In real life operation, such an autonomous system might perform abrupt adjustments to maintain its distance from dangers/obstacles, therefore sacrificing the comfort of the passengers.

A 90-degree manoeuvre performance comparison between a human operator and the ASV is shown in Figure 15. It can be observed that as the vessel made a 90-degree right turn (positive angle), the human operator carefully adjusted the response of the rudder accordingly, which resulted in a more relaxed cornering manoeuvre. As of the ANN model, in order to compensate with the probability to drift during cornering manoeuvre, the autonomous system can be seen as attempting to actively controlling its steering response to maintain its distance to the vertical bank of the river.

The comparative assessment discussed above between a human operator, and the autonomous system has raised a discussion with regard to the element of accuracy bias and comfort in vessel handling. For a human operator, comfort factor in handling is very important to maintain longer endurance of work; however, for an autonomous system, accuracy is more important to reduce the probability of hitting an obstacle as trained in the experimental setup. It can be recommended that such an element of bias and comfort can serve as the potential works in the future. 


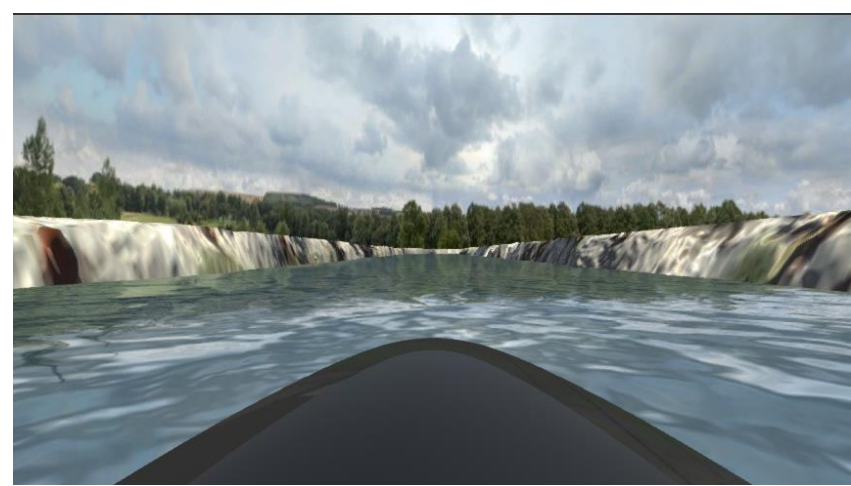

Figure 13. Straight Line Manoeuvre - Human Operator view.

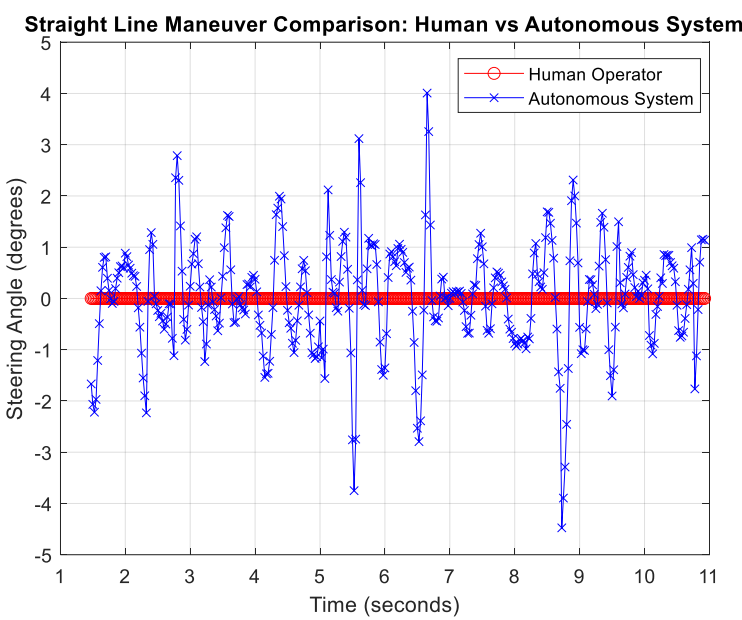

Figure 14. Straight Line Manoeuvre - rudder angle comparison between a Human Operator and Autonomous System.

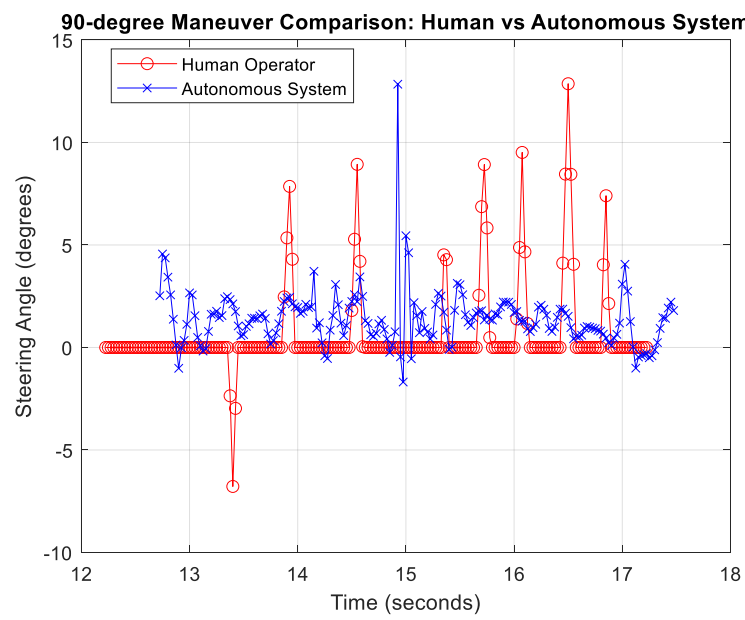

Figure 15. 90-Degree Manoeuvre - rudder angle comparison between a Human Operator and Autonomous System.

\section{CONCLUSIONS}

Presented in this work are the state-of-the-art review and experimental analysis of the use of neuroevolutionary methods in ship design discipline, particularly within the scope of autonomous handling scenarios. Although autonomous vehicles have been progressing rapidly for the land-based vehicles, the research for self-driving in restricted waters (riverine and ports) still possess a significant gap despite its economic and safety impacts. In this work, an illustrative example has been presented using a simplified ship model in a restricted water scenario (vertical riverbank) which reveals that the preliminary neuroevolutionary model is not only good for navigation in restricted water but also capable for avoiding obstacle within the proximity of the distance sensors. Using the end-to-end unsupervised reinforcement learning, the artificial neural network can predict the best steering angle and throttle responses while avoiding collision with other floating objects and riverbanks. Additionally, the handling performance of the ship has been compared between a human operator and the autonomous system (ANN model) The future works may include the consideration of comfort factor in ship handling within the ANN training to ensure that the ASV is not only capable of performing a safe manoeuvre operation, but also comfortable handling for human passengers.

\section{ACKNOWLEDGEMENTS}

The work presented in this research article is funded by Universiti Malaysia Terengganu (Research Intensified Grant Scheme, RIGS, Grant Number: 55192/12) under the theme of Technology \& Engineering (Infrastructure and Transportation).

\section{REFERENCES}

[1] Y. A. Ahmed and K. Hasegawa, "Implementation of automatic ship berthing using artificial neural network for free running experiment," in IFAC Proceedings Volumes (IFAC-PapersOnline), 2013, doi: 10.3182/20130918-4-JP-3022.00036.

[2] Y. A. Ahmed and K. Hasegawa, "Artificial neural network based automatic ship berthing combining PD controlled side thrusters - A combined controller for final approaching to berth," in 2014 13th International Conference on Control Automation Robotics and Vision, ICARCV 2014, 2014, doi: 10.1109/ICARCV.2014.7064504.

[3] K. Priandana, B. Kusumoputro, and E. T. Rahardjo, "The design of ISM-band radar antenna for small boat's trajectory tracking," in QiR 2017 - 2017 15th International Conference on Quality in Research (QiR): International Symposium on Electrical and Computer Engineering, 2017, doi: 10.1109/QIR.2017.8168460.

[4] A. Dabrowski, S. Busch, and R. Stelzer, "A Digital Interface for Imagery and Control of a Navico/Lowrance Broadband Radar," in Robotic Sailing, 2011.

[5] T. Wu, Y. Dong, Z. Dong, A. Singa, X. Chen, and Y. Zhang, "Testing Artificial Intelligence System Towards Safety and Robustness: State of the Art," IAENG Int. J. Comput. Sci., vol. 47, no. 3, 2020.

[6] N. Uddin, "A Two-Wheeled Robot Trajectory Tracking Control System Design Based on Poles Domination Approach," IAENG Int. J. Comput. Sci., vol. 47, no. 2, 2020.

[7] H. Noshahri, T. J. A. De Vries, and J. Van Amerongen, "Towards Automatic Steering of Underactuated Ships," in IFAC-PapersOnLine, 2019, doi: 10.1016/j.ifacol.2019.12.293 
[8] S.-D. Lee, C.-Y. Tzeng, and W.-W. Huang, "Ship Steering Autopilot Based on ANFIS Framework and Conditional Tuning Scheme," Mar. Eng. Front., 2013.

[9] L. Moreira and C. Guedes Soares, "Recursive neural network model of catamaran manoeuvring," Trans. $R$. Inst. Nav. Archit. Part A Int. J. Marit. Eng., 2012, doi: 10.3940/rina.ijme.2012.a3.232.

[10] W. Naeem, S. C. Henrique, and L. Hu, "A Reactive COLREGs-Compliant Navigation Strategy for Autonomous Maritime Navigation," IFACPapersOnLine, 2016, doi: 10.1016/j.ifacol.2016.10.344.

[11] M. Kurowski, H. Korte, and B. P. Lampe, "AGaPaS A new approach for search-and-rescue-operations at sea," in IFAC Proceedings Volumes (IFAC-PapersOnline), 2012, doi: 10.3182/20120919-3-IT-2046.00013.

[12] M. Kurowski and B. P. Lampe, "AGaPaS: A new approach for search-and-rescue-operations at sea," Proc. Inst. Mech. Eng. Part M J. Eng. Marit. Environ., 2014, doi: $10.1177 / 1475090213504392$.

[13] Q. Zhang, N. Jiang, Y. Hu, and D. Pan, “Design of Course-Keeping Controller for a Ship Based on Backstepping and Neural Networks," Int. J. e-Navigation Marit. Econ., 2017, doi: 10.1016/j.enavi.2017.06.004.

[14] Y. Wang, S. Chai, and H. D. Nguyen, "Experimental and numerical study of autopilot using Extended Kalman Filter trained neural networks for surface vessels," Int. J. Nav. Archit. Ocean Eng., 2020, doi: 10.1016/j.ijnaoe.2019.11.004.

[15] M. C. Tsou and C. K. Hsueh, "The study of ship collision avoidance route planning by ant colony algorithm," J. Mar. Sci. Technol., 2010.

[16] Y. A. Ahmed and K. Hasegawa, "Automatic ship berthing using artificial neural network based on virtual window concept in wind condition," in IFAC Proceedings Volumes (IFAC-PapersOnline), 2012, doi: 10.3182/20120912-3-BG-2031.00059.

[17] K. Hasegawa, "From Ship manoeuvrability, controllability, captain's model, traffic model to accident and tsunami analysis," in International Workshop on Nautical Traffic Models, 2013.

[18] Y. A. Ahmed and K. Hasegawa, "Consistently Trained Artificial Neural Network for Automatic Ship Berthing Control," TransNav, Int. J. Mar. Navig. Saf. Sea Transp., 2015, doi: 10.12716/1001.09.03.15.

[19] M. Łącki, "Reinforcement Learning in Ship Handling," TransNav Int. J. Mar. Navig. Saf. Sea Transp., 2008.

[20] M. Łacki, "Speciation of population in neuroevolutionary ship handling," in Marine Navigation and Safety of Sea Transportation, 2009.

[21] M. Łącki, "Neuroevolutionary Ship Maneuvering Prediction System," in Information, Communication and Environment, 2015.

[22] V. L. Tran and N. Im, "A study on ship automatic berthing with assistance of auxiliary devices," Int. J. Nav. Archit. Ocean Eng., 2012, doi: 10.3744/JNAOE.2012.4.3.199.

[23] M. Łącki, "Neuroevolutionary approach to colregs ship maneuvers," TransNav, 2019, doi: 10.12716/1001.13.04.06.

[24] M. Lacki, "Indirect Encoding in Neuroevolutionary Ship Handling," TransNav, Int. J. Mar. Navig. Saf. Sea Transp., 2018, doi: 10.12716/1001.12.01.07.

[25] M. Łącki, "Ship course-keeping with neuroevolutionary algorithms," Zesz. Nauk. Akad. Morskiej w Szczecinie, 2018, doi: 10.17402/287.

[26] C. Chen, X. Q. Chen, F. Ma, X. J. Zeng, and J. Wang, "A knowledge-free path planning approach for smart ships based on reinforcement learning," Ocean Eng., 2019, doi: 10.1016/j.oceaneng.2019.106299.

[27] A. Lazarowska, "Research on algorithms for autonomous navigation of ships," WMU J. Marit. Aff., 2019, doi: 10.1007/s13437-019-00172-0.

[28] D. Looije and Y. Koldenhof, "Unmanned ship simulation with real-time dynamic risk index," Zesz. Nauk. Akad. Morskiej w Szczecinie, 2015.

[29] D. A. Oskin, A. A. Dyda, and V. E. Markin, "Neural network identification of marine ship dynamics," in IFAC Proceedings Volumes (IFAC-PapersOnline), 2013, doi: 10.3182/20130918-4-JP-3022.00018.

[30] B. Liu, S. Z. Wang, Z. X. Xie, J. S. Zhao, and M. F. Li, "Ship recognition and tracking system for intelligent ship based on deep learning framework," TransNav, 2019, doi: 10.12716/1001.13.04.01.

[31] Y. Xue, D. Clelland, B. S. Lee, and D. Han, "Automatic simulation of ship navigation," Ocean Eng., 2011, doi: 10.1016/j.oceaneng.2011.10.011.

[32] R. Skulstad, G. Li, H. Zhang, and T. I. Fossen, “A Neural Network Approach to Control Allocation of Ships for Dynamic Positioning," IFAC-PapersOnLine, 2018, doi: 10.1016/j.ifacol.2018.09.481.

[33] L. P. Perera, "Autonomous ship navigation under deep learning and the challenges in colregs," in Proceedings of the International Conference on Offshore Mechanics and Arctic Engineering - OMAE, 2018, doi: 10.1115/OMAE2018-77672.

[34] T. Statheros, G. Howells, and K. McDonald-Maier, "Autonomous ship collision avoidance navigation concepts, technologies and techniques," J. Navig., 2008, doi: $10.1017 / S 037346330700447 X$.

[35] Y. Cheng and W. Zhang, "Concise deep reinforcement learning obstacle avoidance for underactuated unmanned marine vessels," Neurocomputing, 2018, doi: 10.1016/j.neucom.2017.06.066.

[36] A. I. Kozynchenko and S. A. Kozynchenko, "Applying the dynamic predictive guidance to ship collision avoidance: Crossing case study simulation," Ocean Eng., 2018, doi: 10.1016/j.oceaneng.2018.07.012.

[37] X. D. Cheng, Z. Y. Liu, and X. T. Zhang, "Trajectory optimization for ship collision avoidance system using genetic algorithm," in OCEANS 2006 - Asia Pacific, 2006, doi: 10.1109/OCEANSAP.2006.4393976.

[38] J. D. Schaffer, D. Whitley, and L. J. Eshelman, "Combinations of genetic algorithms and neural networks: A survey of the state of the art," in COGANN 1992 - International Workshop on Combinations of Genetic Algorithms and Neural Networks, 1992, doi: 10.1109/COGANN.1992.273950.

[39] X. Yao, "Evolving artificial neural networks," Proc. IEEE, 1999, doi: 10.1109/5.784219.

[40] D. Floreano, P. Dürr, and C. Mattiussi, "Neuroevolution: From architectures to learning," Evolutionary Intelligence. 2008, doi: 10.1007/s12065-0070002-4.

[41] L. Harris, "Unmanned Marine Systems - ASVs, USVs \& Autonomous Boat Control System," 2020. [Online]. Available:

https://www.unmannedsystemstechnology.com/compa ny/autonomous-surface-vehicles-ltd/.

[42] A. Rescec, "Dynamic Water Physics 2," Unity Assets Store, 2020. [Online]. Available: https://assetstore.unity.com/packages/tools/physics/dyn amic-water-physics-2-147990. 